\title{
Model reference adaptive control of a haptic feedback device for improving force performance
}

- Vu Minh Hung

- Trinh Quang Trung

PetroVietnam University (PVU)

(Manuscript Received on July 23 ${ }^{\text {th }}$, 2013; Manuscript Revised January 14 ${ }^{\text {th }}$, 2014)

\begin{abstract}
:
In this paper, a new adaptive control algorithm of a haptic feedback device is analyzed. Forces applied to the haptic device through human hand movements are modeled as disturbances and compensated in the force control action. A model reference adaptive control (MRAC) scheme is proposed to improve force tracking performance. A separate reference model for every DOF is selected to satisfy rising time, settling time, peak time, and overshoot requirements. General adaptive control laws
\end{abstract}

\begin{abstract}
are developed for tuning gains in the control transfer functions based on the reference model and the force sensor and encoder readings in real time. These control gains cover force tracking performance and compensate human hand disturbances while providing robustness to sensor noise. Stability of the control system is shown analytically. Convergence and boundedness of control gains are also shown through experiments.
\end{abstract}

Keywords: Adaptive force control, haptic device, haptic teleoperation, MRAC, masterslave control, human hand, haptic device modeling.

\section{INTRODUCTION}

Haptic feedback devices have many useful applications such as surgical teleoperation systems. In which a surgeon can use the haptic device to operate a surgical robot working with patients. The haptic device can work as a master to provide desired trajectories and forces for a slave robot. Control of haptic feedback devices has become active research areas. The control algorithm should satisfy the objective of accurate force sensing from the desired forces. The user should feel actual forces from the desired forces not those of the structure of the haptic device. Impedance force control and admittance force control are two force control techniques used for haptic devices [1]. The closed loop impedance control may improve the force performances [2-3].

Adaptive control techniques have proven their advantages with uncertain dynamic systems. Adaptive impedance control is used in haptic simulations to improve transparency and stability [4]. Park and Lee [5] developed an adaptive impedance control method for a haptic device to estimate the stiffness and damping of human hand and to improve force performances.

\section{Trang 102}


Human hand and arm interact with a haptic device and may affect the force control performance. Human hand impedance can be modeled as a mass-spring-damper system [6]. The human hand can be defined as an admittance model where the force input generates the motion output [7-8]. This model is constructed with one mass, two springs and two dampers. Human hand and arm should be properly modeled and included in the haptic force control system. Model reference adaptive control (MRAC) is an interesting method to construct stable control systems. Design of MRAC for teleoperation system with output prediction is presented in [9]. Two MRAC are designed for both master and slave devices to estimate time delay and predict output so that the transparency and stability are improved.

This paper extends the preceding works of force control for a haptic device [3]. Force control model of the haptic device including the human hand model is analyzed to investigate the dynamic effect caused by the human hand movements. A new adaptive impedance force control using MRAC is proposed to achieve good force tracking performances as well as compensate human hand disturbances. The reference model is selected as the third order relative one to satisfy requirements of rise time, settling time, peak time, and overshoot of the force tracking. Adaptive feedforward control is also proposed to compensate the dynamic effects caused by the human hand movements.

\section{FORCE CONTROL MODEL}

A 6-DOF haptic device shown in Figure 1 utilizes two 3-DOF parallel structures similar to the 3-DOF Delta structure. These two 3-DOF parallel structures are divided into the upper structure and the lower structure. The end effectors of the upper and lower structures are connected to a steering handle via universal joints. This haptic device has six legs controlled by six gearless DC motors fixed on the base frame. Each leg is made of hollow aluminum to meet the low weight requirement. Two weight balances are attached to the back extension of the two middle legs to minimize the effect of gravity. Each leg is composed of two links connected by two 2-DOF revolute ball bearing joints such that one revolute joint connects two links while the other revolute joint connects the link to the end effector. The haptic device can provide forces up to $30 \mathrm{~N}$ and torque up to $2 \mathrm{Nm}$. The contact forces $F_{c}$ exerted by the user can be measured with two 3-DOF force sensors attached on the end effectors of the haptic device.

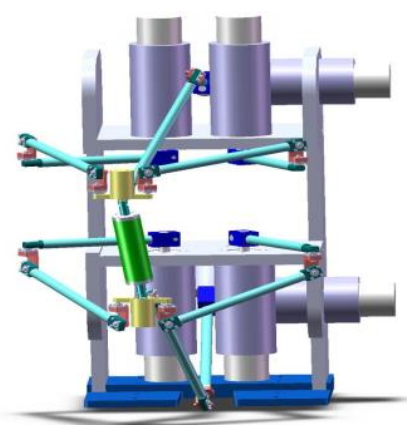

(a) Design model

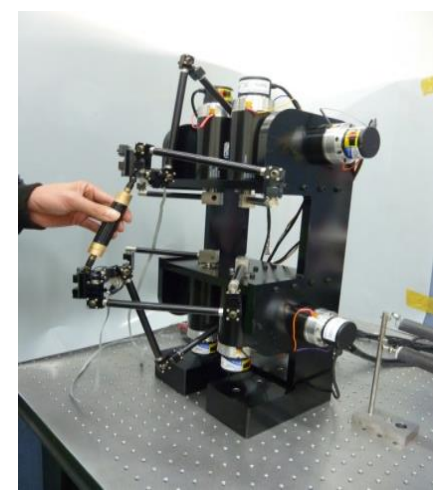

(b) Manufactured model

Fig. 1. A 6-DOF haptic feedback device 
A dynamic equation of a 6-DOF haptic feedback device can be expressed in the Cartesian space as

$$
M\left(x_{h}\right)+V\left(x_{h}, \underset{h}{\&}\right)+G\left(x_{h}\right)=J_{h}^{T} \tau-F_{c}
$$

where $\tau$ is a motor torque vector, $J_{h}$ is Jacobian and $J_{h}^{T} \tau$ is forces generated by motor torques. $M\left(x_{h}\right), V\left(x_{h}, \underset{h}{\&}\right)$, and $G\left(x_{h}\right)$ are inertia matrix, coupling velocity matrix, and gravity force of the haptic device respectively.

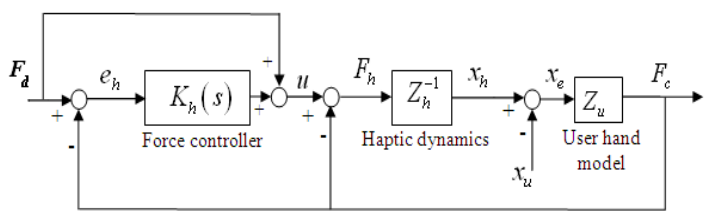

Fig. 2. Control model of a 6-DOF haptic feedback device

$x_{h}=\left[\begin{array}{llllll}x & y & z & \alpha & \beta & \gamma\end{array}\right]^{T}$ is position vector of the steering handle. The contact force $F_{c}$ between the steering handle and the user hand is defined as

$$
F_{c}=B(\not{z}-\underset{u}{\&})+K\left(x_{h}-x_{u}\right)
$$

where $B, K, F_{c}=\left[\begin{array}{llllll}F_{x} & F_{y} & F_{z} & M_{x} & M_{y} & M_{z}\end{array}\right]^{T}$, and $x_{u}=\left[\begin{array}{llllll}\hat{x} & \hat{y} & \hat{z} & \hat{\alpha} & \hat{\beta} & \hat{\gamma}\end{array}\right]^{T}$ are damping matrix, stiffness matrix, contact force vector, and position vector of the user hand respectively. The gravity force $G\left(x_{h}\right)$ can be estimated using a classical dynamic analysis and compensated with feed-forward control action. The dynamic equation is reorganized as

$M\left(x_{h}\right)+V\left(x_{h}, \underset{h}{\&}\right)+G\left(x_{h}\right)=J_{h}^{T} \tau-F_{c}+G\left(x_{h}\right)$
If the estimated gravity force is perfect, the dynamic equation can be shortened as

$$
M\left(x_{h}\right)+V\left(x_{h}, \underset{h}{\&}\right)=J_{h}^{T} \tau-F_{c}
$$

A force control model of haptic device is shown in Figure 2. The relationship between the input force $F_{h}$ to haptic device and its movement $x_{h}$ can be expressed as

$$
F_{h}=Z_{h} x_{h}
$$

The relationship between contact force $F_{c}$ and position errors $x_{e}$ between user hand and haptic device is expressed as

$$
F_{c}=Z_{u} x_{e}
$$

where $x_{e}=x_{h}-x_{u}$. The motor force is $u=J_{h}^{T} \tau$.

The user hand keeps the steering handle of the haptic device and generates the trajectories, $x_{u}$ and $x_{h}$. The user hand can be modeled as a simple 1-DOF mass-spring-damper model [7]. The relationship between the estimated hand trajectory $x_{u}$ and haptic device trajectory $x_{h}$, is expressed as

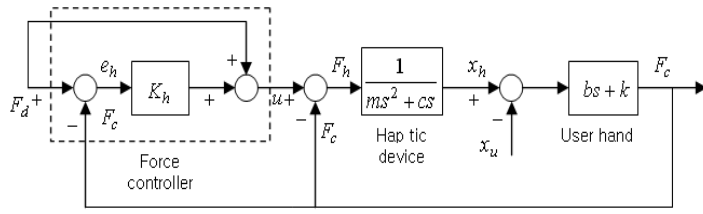

Fig. 3. A 1-DOF force control model of haptic device

$$
\hat{H}=\frac{x_{u}}{x_{h}}=\frac{(b s+k)}{m_{u} s^{2}+\left(b+b_{1}\right) s+\left(k+k_{1}\right)}
$$

Where $b, k$ are the damping and stiffness of user hand and $m_{1}, b_{1}, k_{1}$ are the mass, damping and stiffness of user arm. 
The user hand has nonlinear stiffness and damping since its stiffness and damping change by the grab condition and posture of the arm. The dynamics of 6-DOF haptic device including the user hand can be decoupled under slow movements and represented as a 1-DOF dynamic model.

A simple 1-DOF haptic feedback device is shown in Figure 3. The trajectory $x_{h}$ of haptic device can be determined by encoders while the hand trajectory $x_{u}$ is difficult to measure accurately.

The trajectory of haptic device is used as a desired trajectory for the other slave device such as a slave robot. $F_{d}$ is a desired force for the control system of haptic device. The user hand may feel the force $F_{c}$ as the desired force $F_{d}$ even though the disturbance force from user hand are existed in the system. The error between the desired force $F_{d}$ and feedback force $F_{c}$ is controlled by a force controller $K_{h}$ to supply torques for motors of haptic device.

The closed loop relationship between $F_{d}$ and $F_{c}$ in 1-DOF force model is described as

$$
\begin{aligned}
F_{c}= & \frac{(b s+k)\left(K_{h}+1\right)}{m s^{2}+c s+(b s+k)\left(K_{h}+1\right)} F_{d} \\
& -\frac{(b s+k)\left(m s^{2}+c s\right)}{m s^{2}+c s+(b s+k)\left(K_{h}+1\right)} x_{u}
\end{aligned}
$$

Equation (8) implies that the contact force $F_{c}$ is induced by two inputs of the user hand trajectory $x_{u}$ and the desired force $F_{d}$. Equation (8) can be reformulated as

$$
F_{c}=\frac{(b s+k)\left(K_{h}+1\right)}{m s^{2}+c s+(b s+k)\left(K_{h}+1\right)}\left(F_{d}-F_{u}\right)
$$

where

$$
F_{u}=\frac{\left(m s^{2}+c s\right) x_{u}}{K_{h}+1}=\frac{\left(m s^{2}+c s\right) \hat{H} x_{h}}{K_{h}+1} \approx \frac{\left(m s^{2}+c s\right) x_{h}}{K_{h}+1}
$$

Equation (10) implies that the haptic device dynamic force may be reduced by the feedback control if the control gain of $K_{h}$ is large enough. However, the system becomes unstable if high control gains are selected [10]. The force $F_{u}$ can also be compensated by feedforward control action if the parameters of $\mathrm{m}$ and $\mathrm{c}$ are estimated.

The control objectives in this paper are satisfying good force tracking performance as well as rejecting the undesired dynamic forces caused by the user hand movements. A model reference adaptive control is proposed to satisfy the requirements of force tracking performance criteria. An adaptive feedforward control is also designed to compensate the dynamic force caused by the user hand movements.

\section{MODEL REFERENCE ADAPTIVE CONTROL (MRAC)}

A reference model of a third order relative degree one is selected for the adaptive controller to satisfy requirements of rise time, settling time and overshoot. The reference model is described as

$$
H_{m}=\frac{a_{1} s^{2}+a_{2} s+a_{3}}{s^{3}+a_{4} s^{2}+a_{5} s+a_{3}}=\frac{a_{1}\left(s+z_{1}\right)\left(s+z_{2}\right)}{\left(s+p_{1}\right)\left(s^{2}+2 \xi \omega_{n} s+\omega_{n}^{2}\right)}
$$

where damping ratio $\xi$, natural frequency $\omega_{n}$, a real pole $p_{1}$, two zeros $z_{1}, z_{2}$, and 


$$
\begin{aligned}
& a_{1}=a_{3}\left(z_{1} z_{2}\right)^{-1}, a_{2}=a_{1}\left(z_{1}+z_{2}\right), a_{3}=p_{1} \omega_{n}^{2} \\
& a_{4}=2 \xi \omega_{n}+p_{1}, a_{5}=\omega_{n}^{2}+2 p_{1} \xi \omega_{n},
\end{aligned}
$$

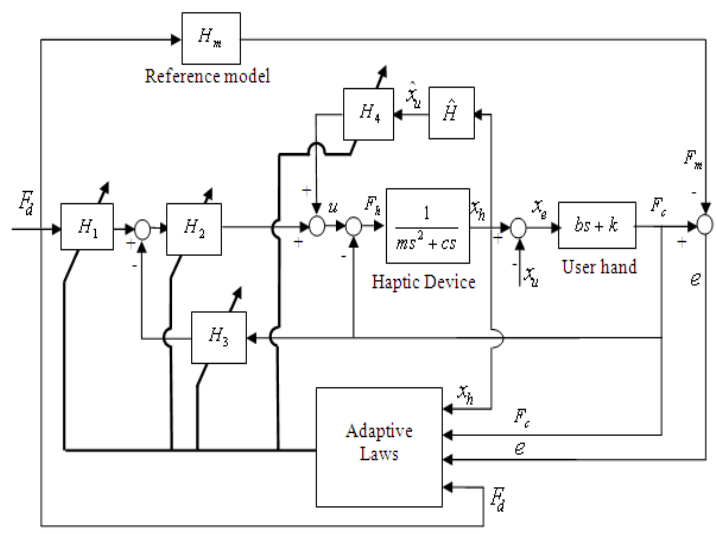

Fig. 4. A diagram of MRAC

The damping ratio and natural frequency determine locations of two complex poles. The damping ratio can be increased to reduce overshoot while the natural frequency is used to adjust settling time. The rise time can be reduced when the pole $p_{1}$ is selected far from the image axis. The overshoot is also controlled by choosing proper zeros. By choosing parameters $\xi, \omega_{n}, p_{1}, z_{1}, z_{2}$ the reference model $H_{m}$ can be obtained to satisfy the requirements of overshoot, settling time, rise time and peak time. In addition the model $H_{m}$ should satisfy requirements of strictly positive real transfer function so that the stability of MRAC will be satisfied.

The designed adaptive force control system using MRAC is shown in Figure 4. MRAC is designed with functions $H_{1}, H_{2}$ and $H_{3}$ to improve force tracking performances, while adaptive feedforward control with a function $\mathrm{H}_{4}$ is designed to compensate dynamic force caused by user hand. The force error between outputs of the reference model and haptic device is used to define control laws to update parameters of $H_{i}, i=1,2,3,4$.

The contact force $F_{c}$ between the user hand and the haptic device is calculated as

$$
F_{c}=\frac{(b s+k) H_{2} H_{1}}{N(s)} F_{d}+\frac{(b s+k)}{N(s)}\left(H_{4} \hat{x}_{u}-\left(m s^{2}+c s\right) x_{u}\right)
$$

where

$N(s)=m s^{2}+(c+b) s+k+(b s+k) H_{3} H_{2}$

Assume that the functions $H_{1}, H_{2}, H_{3}$ are selected to satisfy the perfect tracking as

$$
F_{c}=\frac{(b s+k) H_{2} H_{1}}{N(s)} F_{d}
$$

The function $H_{4}$ should satisfy the following equation.

$$
\frac{(b s+k)}{N(s)}\left(H_{4} \hat{x}_{u}-\left(m s^{2}+c s\right) x_{u}\right)=0
$$

Assume that $\hat{x}_{u} \approx x_{u} \approx x_{h}, H_{4}$ can be selected to eliminate the dynamics of the haptic device such as

$$
H_{4} \approx K_{6} s^{2}+K_{7} s
$$

where $K_{6}=m, K_{7}=c$. However, the parameters of $\mathrm{m}$ and $\mathrm{c}$ are unknown so that control gains $K_{6}, K_{7}$ are adapted to reduce the haptic device dynamics.

The functions $H_{1}, H_{2}, H_{3}$ should be properly selected such that the closed loop transfer function should be equal to the reference model $H_{m} \cdot H_{1}$ is selected as a feedforward gain $K_{1} . H_{2}$ is selected as a second order filter with two adjustable gains $K_{2}$ and $K_{3}$ as

\section{Trang 106}




$$
H_{2}=\frac{1}{1-\frac{K_{2} s+K_{3}}{s^{2}+a_{2} a_{1}^{-1} s+a_{3} a_{1}^{-1}}}
$$

The characteristic equation of second order filter inherits from the numerator of reference model $H_{m}$. The function $H_{3}$ can be selected as a feedback gain $K_{4}$ and a second order filter with an adjustable gain $K_{5}$ as

$$
H_{3}=K_{4}+\frac{K_{5}}{s^{2}+a_{2} a_{1}^{-1} s+a_{3} a_{1}^{-1}}
$$

If parameters of haptic device and user hand are known for the ideal case, the control gains $K_{1}, \ldots, K_{5}$ can be solved. The closed loop transfer function in (14) is calculated as

$$
H_{d c}=\frac{F_{c}}{F_{d}}=\frac{K_{1}(b s+k)\left(a_{1} s^{2}+a_{2} s+a_{3}\right)}{A_{4} s^{4}+A_{3} s^{3}+A_{2} s^{2}+A_{1} s+A_{0}}
$$

\section{Where}

$$
\begin{aligned}
& A_{4}=m a_{1}, A_{3}=m a_{2}+c a_{1}+b a_{1}-m a_{1} K_{2}+b a_{1} K_{4} \\
& A_{2}=m a_{3}+c a_{2}+b a_{2}+a_{1} k-a_{1}(c+b) K_{2}-m a_{1} K_{3}+\left(b a_{2}+k a_{1}\right) K_{4} \\
& A_{1}=c a_{3}+b a_{3}+k a_{2}-k a_{1} K_{2}-a_{1}(c+b) K_{3}+\left(b a_{3}+k a_{2}\right) K_{4}+b a_{1} K_{5} \\
& A_{0}=k a_{3}-k a_{1} K_{3}+k a_{3} K_{4}+k a_{1} K_{5}
\end{aligned}
$$

The closed loop transfer function of system in (19) is compared with the reference model $H_{m}$ to find ideal control gains as

$$
K_{15}=A^{-1} B
$$

where $K_{15}=\left[\begin{array}{lllll}K_{1} & K_{2} & K_{3} & K_{4} & K_{5}\end{array}\right]^{T}$

$$
\begin{aligned}
A & =\left[\begin{array}{llllc}
b a_{4} & 0 & 0 & 0 & 0 \\
b a_{5}+k a_{4} & m a_{1} & 0 & -b a_{1} & 0 \\
b a_{2}+k a_{5} & a_{1}(c+b) & a_{1} m & -b a_{2}-k a_{1} & 0 \\
b a_{3}+k a_{2} & k a_{1} & a_{1}(c+b) & -b a_{3}-k a_{2} & -b a_{1} \\
b a_{4} & 0 & k a_{1} & -k a_{3} & -k a_{1}
\end{array}\right] \\
B & =\left[\begin{array}{l}
m a_{1} \\
m a_{2}+c a_{1}+b a_{1} \\
m a_{3}+c a_{2}+b a_{2}+a_{1} k \\
c a_{3}+b a_{3}+k a_{2} \\
k a_{3}
\end{array}\right]
\end{aligned}
$$

If the system parameters are known, the ideal control law is calculated as

$$
u=\sum_{i=1}^{7} K_{i} Y_{i}=K^{T} Y
$$

where

$$
\begin{aligned}
& Y=\left[Y_{1}, \ldots, Y_{7}\right]^{T}, K^{T}=\left[K_{1}, \ldots, K_{7}\right] \\
& Y_{1}=F_{d}, Y_{2}=\frac{s u}{s^{2}+a_{2} a_{1}^{-1} s+a_{3} a_{1}^{-1}}, Y_{3}=\frac{u}{s^{2}+a_{2} a_{1}^{-1} s+a_{3} a_{1}^{-1}} \\
& Y_{4}=F_{c}, Y_{5}=\frac{F_{c}}{s^{2}+a_{2} a_{1}^{-1} s+a_{3} a_{1}^{-1}}, Y_{6}=s^{2} \hat{x}_{u}, Y_{7}=s \hat{x}_{u}
\end{aligned}
$$

The ideal control law also can be considered in the time domain as

$$
u(t)=\sum_{i=1}^{7} K_{i}(t) Y_{i}(t)=K(t)^{T} Y(t)
$$

If the ideal control law is given, the error $e=F_{c}-F_{m}$ will be zero. The contact force $F_{c}^{*}$ in ideal case then becomes

$$
F_{c}^{*}=F_{m}=H_{m} Y_{1}=H_{m} F_{d}
$$


Since parameters of user hand and haptic device are unknown, the control gains should be updated with an adaptive law. The estimated control law is defined as

$$
\hat{u}(t)=\hat{K}(t)^{T} Y(t)
$$

where $\quad \hat{K}=\left[\hat{K}_{1}, \ldots, \hat{K}_{7}\right]^{T} \quad$ and

$\hat{K}=K+\Delta K, \Delta K$ is the error of estimated control gains. The estimated control law is then reformulated as

$$
\hat{u}=K_{1} \hat{Y}_{1}+\sum_{i=2}^{7} K_{i} Y_{i}
$$

where $\hat{Y}_{1}=Y_{1}+\frac{\Delta K^{T} Y}{K_{1}}$

The contact force $F_{c}$ with parameter uncertainties is then described as

$$
F_{c}=H_{m}\left(Y_{1}+\frac{\Delta K^{T} Y}{K_{1}}\right)
$$

The error between outputs $F_{c}$ and reference model is then

$$
e=F_{c}-F_{c}^{*}=F_{c}-H_{m} Y_{1}=H_{m}\left(\frac{\Delta K^{T} Y}{K_{1}}\right)
$$

If $H_{m}$ is strictly positive real transfer function, the adaptive law can be selected as [11]

$$
\Delta K^{\&}=-\operatorname{sign}\left(K_{1}\right) \gamma e Y
$$

where $\gamma$ is a given positive constant and $K_{1}>0$. The adaptive law is then obtained as

$$
\stackrel{\&}{K}(t)=-\gamma e Y
$$

Substituting the reference model $H_{m}$ to the error dynamic equation (27) leads to

$$
e=\frac{a_{1} s^{2}+a_{2} s+a_{3}}{s^{3}+a_{4} s^{2}+a_{5} s+a_{3}} \frac{\Delta K^{T} Y}{K_{1}}
$$

Equation (30) can be expressed by a state space equation as

$$
\begin{aligned}
& X_{e}^{\&}=A_{e} X_{e}+B_{e} \frac{\Delta K^{T} Y}{K_{1}} \\
& e=C_{e}^{T} X_{e}
\end{aligned}
$$

where

$$
\begin{gathered}
X_{e}=\left[\begin{array}{l}
\& \\
y
\end{array}\right], A_{e}=\left[\begin{array}{ccc}
-a_{4} & -a_{5} & -a_{3} \\
1 & 0 & 0 \\
0 & 1 & 0
\end{array}\right], B_{e}=\left[\begin{array}{l}
1 \\
0 \\
0
\end{array}\right], C_{e}=\left[\begin{array}{l}
a_{1} \\
a_{2} \\
a_{3}
\end{array}\right] \\
y=\frac{1}{s^{3}+a_{4} s^{2}+a_{5} s+a_{3}} \frac{\Delta K^{T} Y}{K_{1}}
\end{gathered}
$$

The reference model $H_{m}$ is selected to satisfy the requirements of strictly positive real transfer function. The Kalman-Yakubovich lemma [11] indicates that there exists symmetric positive matrix $\mathrm{P}$ and $\mathrm{Q}$ so that the following equation is satisfied.

$$
\begin{aligned}
& A_{e}^{T} P+P A_{e}=-Q \\
& P B_{e}=C_{e}
\end{aligned}
$$

A Lyapunov function of $X_{e}, \Delta K$ is selected as

$$
V=X_{e}^{T} P X_{e}+\frac{1}{\gamma K_{1}} \Delta K^{T} \Delta K
$$

Taking its derivative to obtain

$$
\mathscr{L}=-X_{e}^{T} Q X_{e} \leq 0
$$

Therefore the dynamic system of force error is stable and $\Delta K, X_{e}$ are bounded, so $e=C_{e} X_{e}$ is also bounded. If $Y$ is bounded then $X_{e}^{\&}$ is bounded and $-X_{e}^{T} Q X_{e}^{\&} \leq 0$ is also bounded. Thus conditions of Barbalet's lemma 
are satisfied. This means that $\mathbb{V}_{\text {goes }}$ to zeros when time goes to infinity. $e=C_{e} X_{e}$ then converges to zeros.

\section{EXPERIMENTS}

A digital controller dSPACE1103 is used to implement control algorithms. The contact forces $F_{c}$ are measured by two 3-DOF force sensors on the haptic device. $F_{d}$ is the desired force to MRAC while the feedback force is $F_{c}$ from the user hand. The output $u$ of MRAC is the force command to the haptic device so it is converted into required torques by inverse transposed

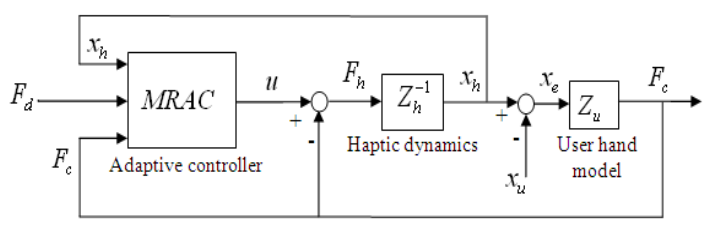

Fig. 5. An adaptive force control model of haptic device

of Jacobian matrix. Six components of desired force $F_{d}$ require six model reference adaptive controllers separately. The haptic control system using MRAC is shown in Figure 5.

Two different reference models are obtained for moment and force components because of different dynamic effects. A reference model for forces has rise time of $0.02 \mathrm{sec}$, settling time of $0.2 \mathrm{sec}$, and overshoot of $1.5 \%$ as

$$
H_{m}=\frac{33(s+24)(s+25)}{(s+22)\left(s^{2}+54 s+900\right)}
$$

The reference model for moments has rise time of $0.09 \mathrm{sec}$, settling time of $0.15 \mathrm{sec}$, and overshoot of $0 \%$ as

$$
H_{m}=\frac{23(s+25)(s+30)}{(s+22)\left(s^{2}+50.4 s+784\right)}
$$

The transfer function $\hat{H}$ is selected to obtain reasonable errors between the user hand trajectory and haptic device trajectory as

$$
\hat{H}=\frac{20 s+200}{0.8 s^{2}+25 s+205}
$$

The closed loop force control algorithm using MRAC was developed and implemented in the digital controller.

The MRAC for step forces of the haptic device was first tested in order to evaluate the reduction of dynamic effects such as frictions, inertia and gravity. The desired forces $\mathrm{F}_{\mathrm{x}}$ of $5 \mathrm{~N}$, $F_{y}$ and $F_{z}$ of $3 N$ are applied to the haptic device while the user hand generates shaking motions working as external disturbances. The forces performances are shown in Figure 6 indicate that the contact forces $F_{c}$ of haptic feedback device can track the desired force $F_{d}$. The control gains for $F_{x}$ in Figure 7 can be converged into certain values so the force errors can be reduced to zeros.

The sine force experiments of MRAC are shown in Figure 8. The comparison indicates that the forces of the haptic device tracked those of desired sine forces well. However, there are small force errors along to $\mathrm{Fz}$-axis because of gravity effects. Control gains of MRAC are converged and bounded as shown in Figure 9. 

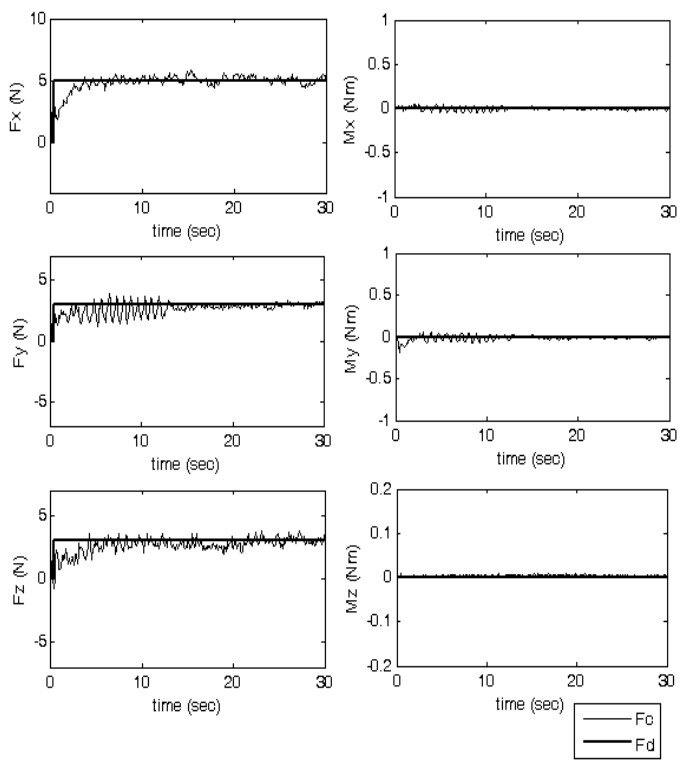

Fig. 6. Step force responses of a haptic device with MRAC
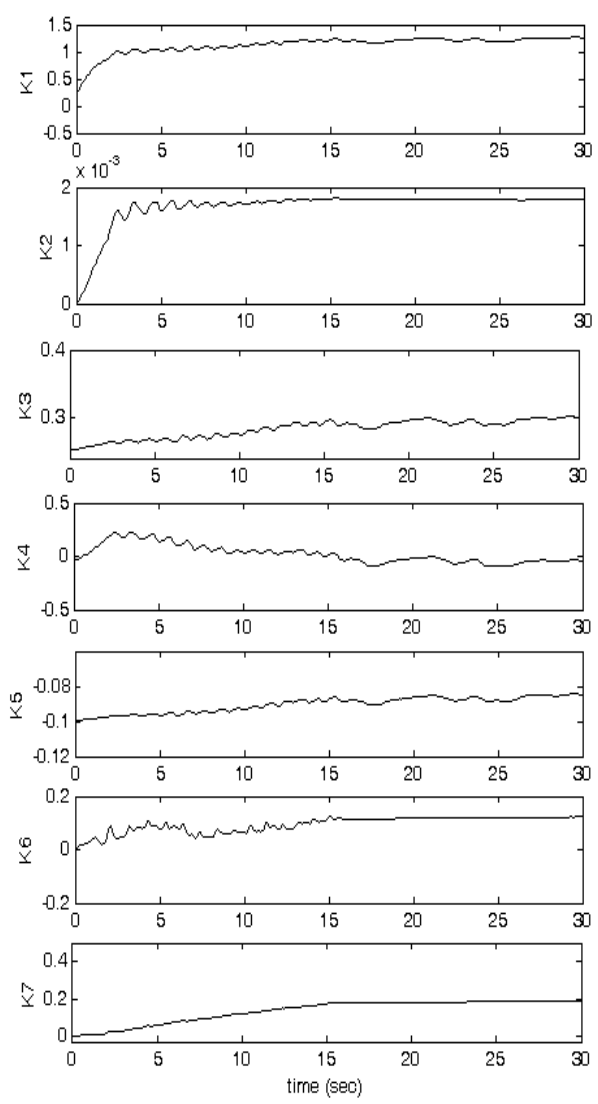

Fig. 7. Control gains of MRAC for step force $F_{x}$
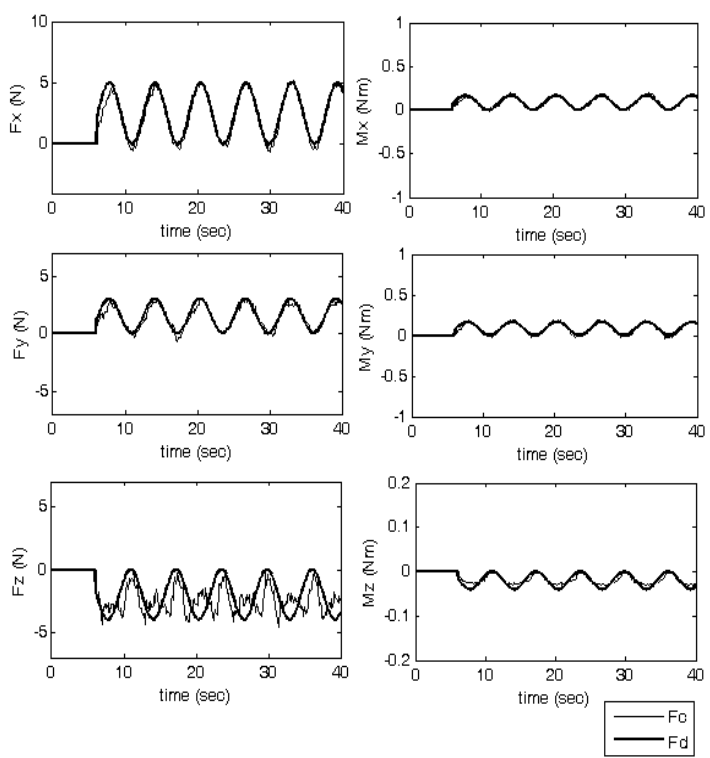

Fig. 8. Sine force responses of a haptic device with MRAC
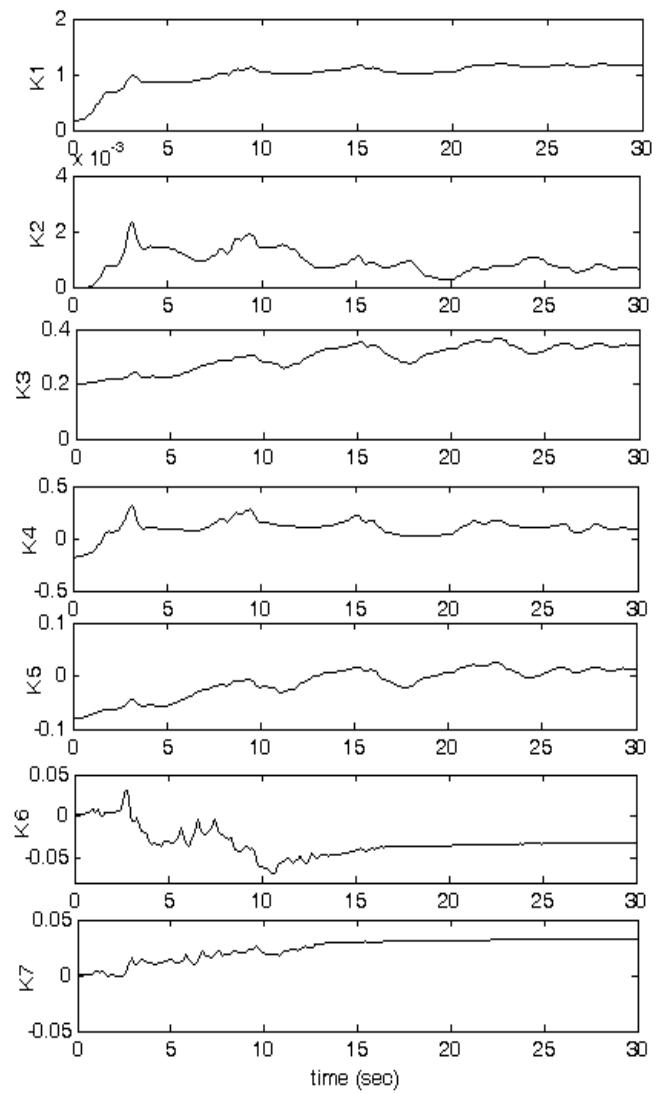

Fig. 9. Control gains of MRAC for sine force $F_{x}$

\section{Trang 110}


Experiments of MRAC indicate that the control gains are converged to certain values. This implies the control system is stable and the force error is bounded.

However, if the desired force $F_{d}=0$, the output of reference model $F_{m}=0$. The adaptive law is recalculated as

$$
\stackrel{\&}{K}=-\gamma e Y=-\gamma F_{c} Y
$$

The control gain $\stackrel{\&}{K}$ is updated as

$$
\stackrel{\&}{K_{4}}=-\gamma e F_{c}=-\gamma F_{c}^{2} \leq 0
$$

This means the control gain keeps decreasing until the contact force equals zeros. It is impossible to eliminate $100 \%$ error in the real-time control system because of noises from force sensors. Therefore, this problem leads to higher control gains and bad feeling forces on the user hand.

In order to improve robustness of adaptive control, projection method in [12] can be applied to limit the control gain. Thus the adaptive law can be modified as

$$
K_{4}^{\&}= \begin{cases}-\gamma e F_{c} & \text { if } \hat{K}_{4} \geq K_{\min } \\ 0 & \text { othewise }\end{cases}
$$

Trajectories and forces of haptic device for free movements $\left(F_{d}=0\right)$ are shown in Figure 10 and 11. The control gains in Figure 12 are updated to reduce contact force $F_{c}$ caused by dynamics of haptic device and user hand. The control gain $\hat{K}_{4}$ decreases until it hits a limitation. This projection technique helps to improve feeling on user hand and keep the stability of system in free movements.
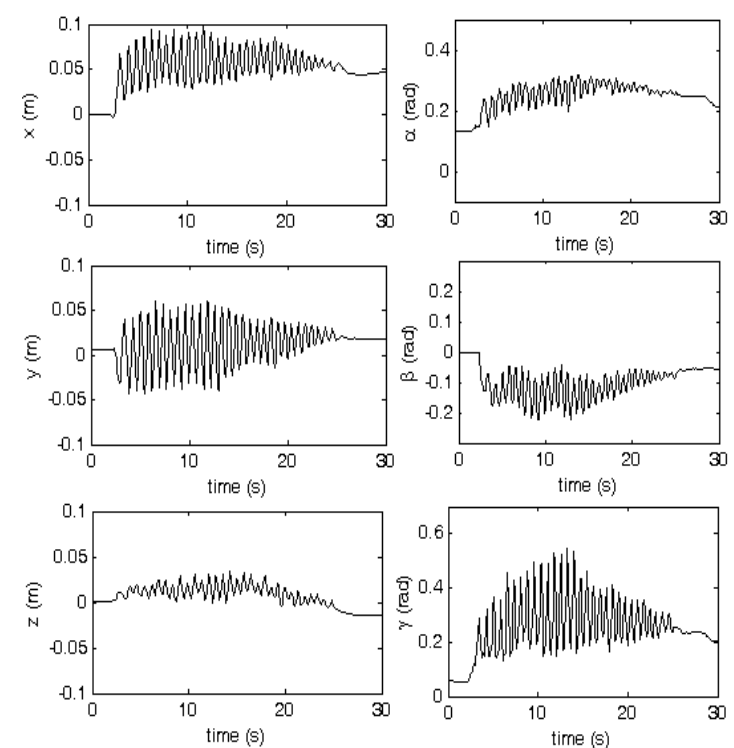

Fig. 10. Trajectory of haptic device for free movement
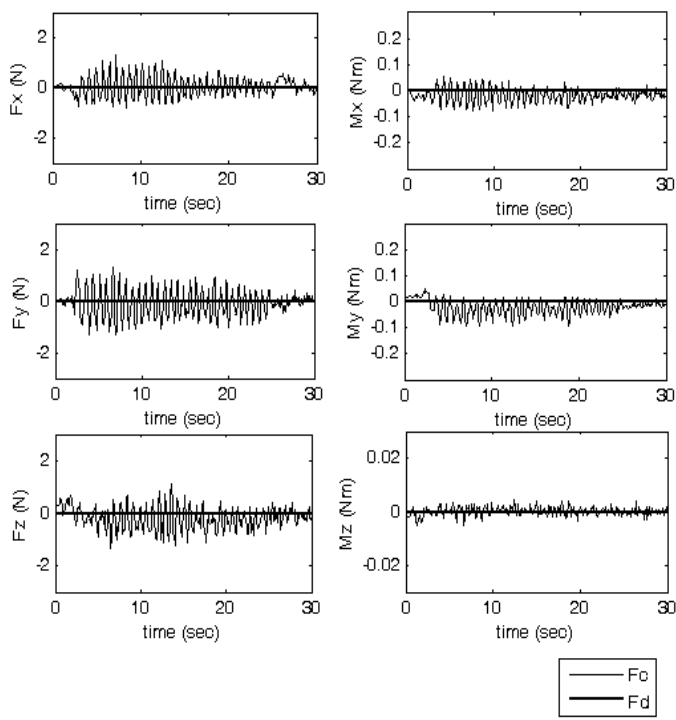

Fig. 11. Contact force of haptic device for free movement

\section{CONCLUSIONS}

This paper presents a new adaptive force control for the haptic feedback device. A model reference adaptive control using MRAC is designed to satisfy good force tracking 
performances as well as to reject the undesired dynamic forces caused by the user hand movements.

The new MRAC is designed with seven control gains in which feedforward and feedback gains $\left(\mathrm{K}_{1}, \mathrm{~K}_{4}\right)$ help to improve tracking performances while filter gains $\left(\mathrm{K}_{2}, \mathrm{~K}_{3}\right.$, $\left.\mathrm{K}_{5}\right)$ and trajectory gains $\left(\mathrm{K}_{6}, \mathrm{~K}_{7}\right)$ reduce noises of sensor and the dynamic effects of user hand respectively. All control gains work to improve force performances.
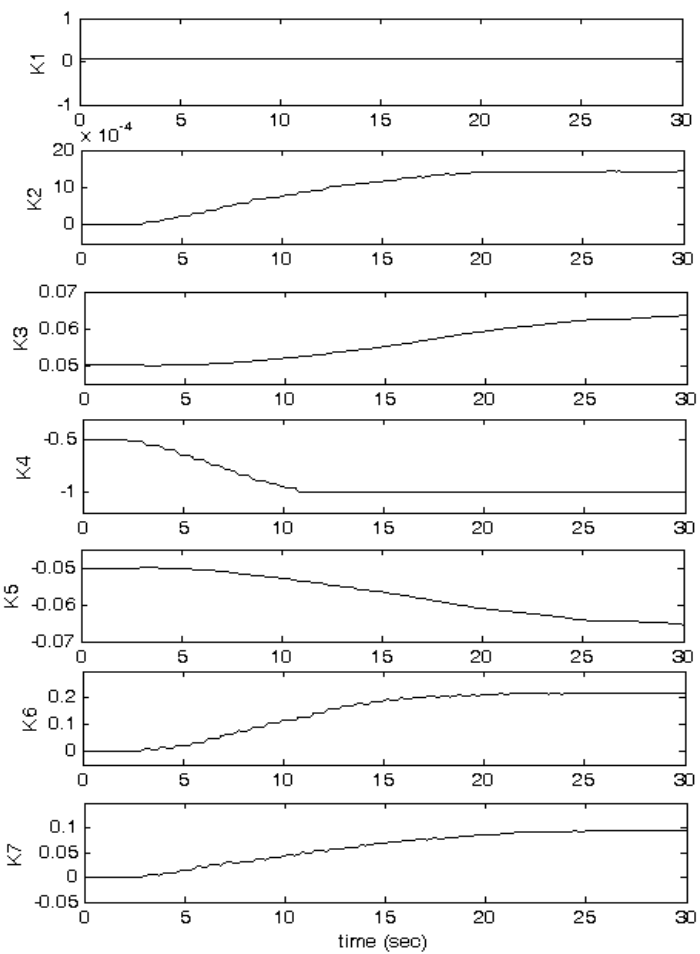

Fig.12. Control gains of MRAC for $F_{x}$ for free movement

The reference model is selected as the third order with relative degree one to satisfy high requirements of rise time, settling time and overshoot. The stability and tracking convergence are proved based on Lyapunov and Barbalat's lemmas. Experiments of haptic feedback device show good performances of MRAC in a manner that force tracking is improved.

This control algorithm can be used for surgical robot teleoperation or master-slave systems. 


\section{Điều khiển thích nghi dựa theo mô hình cho thiết bị phản hồi xúc giác để cải thiện lực bám}

- Vũ Minh Hùng

- Trịnh Quang Trung

Trường Đại Học Dầu Khí Việt Nam (PVU)

\section{TÓM TÁ́T :}

Bài báo này trình bày một thuật toán điều khiển thích nghi lực cho thiết bị phản hồi xúc giác 6 bậc tự do. Lực của tay người khi cầm nắm thiết bị phản hồi xúc giác sẽ được mô hình hóa như nhiễu ngoài tác động vào hệ thống điều khiển. Để làm giảm ảnh hưởng của nhiễu ngoài và tăng khả năng bám của lực thì bộ điều khiển thích nghi dựa theo mô hình (MRAC) được sử dụng. Mô hình mẫu được lựa chọn để phù hợp với đặc tính động lực học của từng loại bậc tự do dịch chuyển tịnh tiến hoặc quay. Luật điều khiển thích nghi được thiết kế để thay đổi tham số điều khiển

theo thời gian thực dựa trên mô hình mẫu, tín hiệu cảm biến lực và encoder động cơ. Thuật toán MRAC này sẽ làm giảm ảnh hưởng của nhiễu ngoài từ tay người và nhiễu từ cảm biến đo, từ đó làm cho lực cầm nắm thiết bị phản hồi xúc giác bám theo lực mong muốn được tốt hơn. Sự ổn định và hội tụ của thuật toán MRAC cũng được chứng minh trên lý thuyết và kiểm chứng bằng thực nghiệm. Kết quả thực nghiệm với lực mong muốn dạng Step và Sine đều cho thấy lực phản hồi bám rất tốt và các tham số điều khiển hội tụ.

Từ khóa: Điều khiển thích nghi, mô hình hóa robot, điều khiển MRAC, điều khiển lực, thiết bị phản hồi xúc giác.

\section{REFERENCES}

[1]. A. Abdossalami and S. Sirouspour, "Adaptive control of haptic interaction with impedance and admittance type", IEEE proceeding, Symposium on Haptic Interfaces for Virtual Environments and Teleoperator Systems, Nevada, USA, March, 2008, pp. 145-152.

[2]. Vu Minh Hung and Uhn Joo Na, "Teleoperation of a 6-DOF serial robot using a new 6-DOF haptic interface," in IEEE Int. Symp. on Haptic Audio-Visual, Environments and Games, Phoenix, Arizona, USA, Oct., 2010, pp. 1-6.

[3]. Vu Minh Hung and Uhn Joo Na, "A new 6DOF haptic device for Tele-operation of a 6-DOF serial robots," in IEEE Transaction on Instrumentation and Measurements, vol. 60 , no. $11,2011$. 
[4]. A. Abdossalami and S. Sirouspour, "Adaptive Control for Improved Transparency in Haptic Simulations" IEEE Transaction on haptics, vol. 2, no. 1, 2009.

[5]. H. Park and J. M. Lee, "Adaptive impedance control of a haptic interface", Mechatronics, vol. 14, 2004, pp. 237-253.

[6]. T. Tsuji, P. Morasso, K. Goto, K. Ito, "Human hand impedance characteristics during maintained posture", Biol. Cybern. vol.72, pp. 475-485, 1995.

[7]. J. E. Speich, L. Shao, M. Goldfarb, "Modeling the human hand as it interacts with a telemanipulation system", Mechatronics vol. 15, pp. 1127-1142, 2005.

[8]. H. S. Woo and D. Y. Lee, "Exploitation of the Impedance and Characteristics of the Human Arm in the Design of Haptic Interfaces, " IEEE Transaction on Industrial Electronics, vol. 58, no. 8, pp. 3221-3233, 2011.
[9]. K. H. Suny and H. Momeni, "Model reference adaptive control design for a teleoperation system with output prediction", J Intell Robot Syst, vol. 59, 2010, pp. 319-339.

[10]. J. J. Gil, A. Rubio and J. Savall, "Decreasing the apparent inertia of an

[11]. impedance haptic device by using force feedforward," IEEE Trans.

[12]. Control System and Tech, vol. 17, no. 4, pp. 833-838, 2009.

[13]. Jean-Jacques, E. Slotine and Weiping Li, “Applied nonlinear control”, 1991.

[14]. Petros A. Ioannou and Jing Sun, "Robust adaptive control", 1996. 